\title{
Air pollution and subclinical interstitial lung disease: the Multi-Ethnic Study of Atherosclerosis (MESA) air-lung study
}

\author{
Coralynn Sack ${ }^{1}$, Sverre Vedal ${ }^{1,2,3}$, Lianne Sheppard ${ }^{2,4}$, Ganesh Raghu $^{5}$, \\ R. Graham Barr ${ }^{6,7}$, Anna Podolanczuk ${ }^{6}$, Brent Doney ${ }^{8}$, Eric A. Hoffman?, \\ Amanda Gassett ${ }^{2}$, Karen Hinckley-Stukovsky ${ }^{4}$, Kayleen Williams ${ }^{10}$, \\ Steve Kawut ${ }^{11}$, David J. Lederer (1) 6,7,12 and Joel D. Kaufman ${ }^{2,3,12}$
}

Affiliations: ${ }^{1}$ Dept of Medicine, University of Washington, Seattle, WA, USA. ${ }^{2}$ Dept of Environmental and Occupational Health Sciences, University of Washington, Seattle, WA, USA. ${ }^{3}$ Dept of Epidemiology, University of Washington, Seattle, WA, USA. ${ }^{4}$ Dept of Biostatistics, University of Washington, Seattle, WA, USA. ${ }^{5}$ Dept of Medicine, Center for Interstitial Lung Diseases, University of Washington Medical Center, Seattle, WA, USA. ${ }^{6}$ Dept of Medicine, Columbia University Medical Center, New York, NY, USA. 7 Dept of Epidemiology, Columbia University Medical Center, New York, NY, USA. ${ }^{8}$ Respiratory Health Division, National Institute for Occupational Safety and Health, Centers for Disease Control and Prevention, Morgantown, WV, USA. 'Dept of Radiology, Carver School of Medicine, University of lowa, lowa City, IA, USA. ${ }^{10}$ Collaborative Health Studies Coordinating Center, University of Washington, Seattle, WA, USA. ${ }^{11}$ Depts of Medicine and Epidemiology, Perelman School of Medicine, University of Pennsylvania, Philadelphia, PA, USA. ${ }^{12}$ Both authors contributed equally.

Correspondence: David J. Lederer, Room 3-321A, Columbia University Medical Center, 161 Fort Washington Avenue, New York, NY 10032, USA. E-mail: dl427Acumc.columbia.edu

\section{@ERSpublications}

Exposure to ambient air pollution was associated with qualitative and quantitative measurements of subclinical ILD http://ow.ly/5OfU30gt2q7

Cite this article as: Sack C, Vedal S, Sheppard L, et al. Air pollution and subclinical interstitial lung disease: the Multi-Ethnic Study of Atherosclerosis (MESA) air-lung study. Eur Respir J 2017; 50: 1700559 [https://doi.org/10.1183/13993003.00559-2017].

ABSTRACT We studied whether ambient air pollution is associated with interstitial lung abnormalities (ILAs) and high attenuation areas (HAAs), which are qualitative and quantitative measurements of subclinical interstitial lung disease (ILD) on computed tomography (CT).

We performed analyses of community-based dwellers enrolled in the Multi-Ethnic Study of Atherosclerosis (MESA) study. We used cohort-specific spatio-temporal models to estimate ambient pollution (fine particulate matter ( $\mathrm{PM} 2.5)$, nitrogen oxides $\left(\mathrm{NO}_{\mathrm{x}}\right)$, nitrogen dioxide $\left(\mathrm{NO}_{2}\right)$ and ozone $\left(\mathrm{O}_{3}\right)$ ) at each home. A total of 5495 participants underwent serial assessment of HAAs by cardiac CT; 2671 participants were assessed for ILAs using full lung CT at the 10-year follow-up. We used multivariable logistic regression and linear mixed models adjusted for age, sex, ethnicity, education, tobacco use, scanner technology and study site.

The odds of ILAs increased 1.77-fold per $40 \mathrm{ppb}$ increment in $\mathrm{NO}_{\mathrm{x}}$ (95\% CI 1.06 to 2.95, $\mathrm{p}=0.03$ ). There was an overall trend towards an association between higher exposure to $\mathrm{NO}_{\mathrm{x}}$ and greater progression of HAAs $\left(0.45 \%\right.$ annual increase in HAAs per $40 \mathrm{ppb}$ increment in $\mathrm{NO}_{\mathrm{x}} ; 95 \% \mathrm{CI}-0.02$ to $0.92, \mathrm{p}=0.06)$. Associations of ambient fine particulate matter $(\mathrm{PM} 2.5), \mathrm{NO}_{\mathrm{x}}$ and $\mathrm{NO}_{2}$ concentrations with progression of HAAs varied by race/ethnicity $(\mathrm{p}=0.002,0.007,0.04$, respectively, for interaction) and were strongest among non-Hispanic white people.

We conclude that ambient air pollution exposures were associated with subclinical ILD. 


\section{Introduction}

Air pollution is a risk factor for adverse respiratory outcomes. Many studies have shown associations between pollution exposure and the incidence and/or progression of multiple pulmonary diseases, including asthma [1], chronic obstructive pulmonary disease (COPD) [2], bronchiectasis [3], respiratory infections [4], chronic lung allograft dysfunction [5] and lung cancer [6]. There has been little research on whether air pollution contributes to the aetiology or accelerates the progression of interstitial lung diseases (ILDs), a heterogeneous group of chronic lung diseases characterised by inflammation and fibrosis of the pulmonary parenchyma that affect nearly one out of 200 older adults in the USA [7].

ILD is often diagnosed after the onset of irreversible parenchymal fibrosis. Early symptoms are nonspecific and frequently attributed to other chronic pulmonary or cardiac diseases. Owing to the late presentation of clinical manifestations and the presumed long latency between exposure and disease development, the underlying aetiologies of many of the ILDs are unknown.

The growing recognition and identification of subclinical forms of ILD on computed tomography (CT) provides a unique opportunity to investigate antecedent causes, rather than consequences, of ILD. Interstitial lung abnormalities (ILAs), a visual assessment of early interstitial changes in nondependent portions of the lung, are a well-validated qualitative measurement of subclinical ILD [8]. A quantitative CT attenuation-based phenotype of subclinical ILD, termed high attenuation areas (HAAs), has also been shown to be a marker of subclinical lung inflammation and fibrosis [9-11].

Longitudinal studies estimate that $20-46 \%$ of participants with ILAs have progressive imaging abnormalities, with approximately $4 \%$ developing features of usual interstitial pneumonia (UIP) [12]. Even in the absence of clinical ILD, populations with subclinical ILD have more respiratory symptoms, physiologic decrements and higher mortality $[10,13,14]$.

Inhalational exposures underlie the pathobiology of asbestosis, hypersensitivity pneumonitis and smoking-related ILDs. Ambient air pollutants may initiate or contribute to the cycle of alveolar injury, disordered repair and fibrosis seen in the ILDs, and have been associated with clinical exacerbations in interstitial fibrosis patients [15]. Air pollutants, including fine and ultrafine particles and traffic-related gases, can deposit throughout the airways and alveoli, causing either direct damage or triggering inflammation and oxidative stress. This could potentially result in alveolar cell injury or senescence, endothelial dysfunction, mononuclear cell interstitial inflammation and fibroblast proliferation.

The association between air pollutants and ILD has not been previously investigated in a population-based study. We hypothesised that exposure to air pollution would be associated with qualitative and quantitative measurements of subclinical ILD in community-dwelling adults.

This article has supplementary material available from erj.ersjournals.com

Received: March 172017 | Accepted after revision: Sept 012017

Support statement: MESA and work in this manuscript was supported by the National Heart, Lung, and Blood Institute (NHLBI) through the following grants and contracts: N01-HC-95159, N01-HC-95160, N01-HC-95161, N01-HC-95162, N01-HC-95163, N01-HC-95164, N01-HC-95165, N01-HC-95166, N01-HC-95167, N01-HC-95168, N01-HC-95169, T32HL007287; by the National Center for Research Resources (NCRR) through UL1-TR-000040, UL1-TR-001079, R01 HL103676, R01 HL077612, R01 HL093081, RC1 HL100543 and K24 HL131937; and by the National Institute of Environmental Health Sciences through P50ES015915, P30ES07033, P30ES007033 and K24ES013195. This research has also been supported by a grant from the US Environmental Protection Agency's (EPA's) Science to Achieve Results (STAR) programme and the publication was developed under Assistance Agreement RD831697 awarded by the EPA to the University of Washington (WA, USA). This work was also funded by the Pulmonary Fibrosis Foundation and the Rocco Guinta Research Fund. Funding information for this article has been deposited with the Crossref Funder Registry. Role of the funding source: The study was overseen by a Steering Committee and an External Scientific Advisory Committee (ESAC). The NHLBI, National Institute for Occupational Safety and Health (NIOSH), EPA, NCRR, Rocco Guinta Fund, and Pulmonary Fibrosis Foundation had no role in the collection, analysis, or interpretation of the data, the writing of the report, or the decision to submit the paper for publication. All authors were provided with complete access to the data and all authors share responsibility for the decision to submit the manuscript.

Disclaimer: This report has not been formally reviewed by the Environmental Protection Agency (EPA). The findings and conclusions in this report are those of the authors and do not necessarily represent the views of the EPA or NIOSH. The EPA and NIOSH do not endorse any products or commercial services mentioned in this publication.

Conflict of interest: Disclosures can be found alongside this article at erj.ersjournals.com 


\section{Methods}

Study design and participants

The Multi-Ethnic Study of Atherosclerosis (MESA) is a prospective cohort study funded by the National Heart Lung and Blood Institute to investigate subclinical cardiovascular disease. MESA and ancillary studies, MESA Lung and MESA Air, are described in depth elsewhere and served as the sampling frame for this study $[10,16]$. Informed consent was obtained for all participants and the study was approved by the Institutional Review Board at collaborating centres.

Briefly, MESA enrolled 6814 participants, aged 45-84 years from six centres around the USA: Baltimore, MD; Chicago, IL; Los Angeles County, CA; New York City, NY; St Paul, MN; and Winston Salem, NC. Participants were recruited between 2000 through 2002, and underwent questionnaires regarding demographics, family history, medical history and lifestyle habits. Between 2005 and 2007, MESA Air recruited an additional 257 participants from Rockland County, NY, Riverside County, CA and Los Angeles County, LA. By study design, all participants were free of known cardiovascular disease at the start of the study; there were no selection criteria based on lung disease, respiratory symptoms or smoking history. At enrolment and during the four subsequent examinations, participants had noninvasive assessment of cardiovascular status including cardiac CT scans, which image approximately $70 \%$ of the lung volume from main carina to lung. By design, all returning participants had a repeat cardiac CT scan at either examination 2 or 3 , and $30 \%$ of the cohort had a third cardiac CT scan at examination 4. An additional subset of 3200 participants underwent cardiac CT scan again at examination 5, during years 2010-12, nearly all of whom had a full lung CT scan at this time.

The sampling scheme of participants that were included in our study is described in detail in the supplementary material.

\section{Interstitial lung abnormalities}

Full lung MESA CT scans were acquired in years 2010-12 (10-year follow-up) at suspended full inspiration using the MESA Lung/SPIROMICS protocol and images were reconstructed using $0.625-\mathrm{mm}$ slice thickness [17]. One of five board-certified radiologists (inter-reader kappa 0.47 [0.14-0.80]) reviewed the full lung CT scans for ILAs, which was defined as any scan read as "definite" ILD or "suspicious" for ILAs. "Definite" ILD was defined as bilateral fibrosis in multiple lobes associated with honeycombing and traction bronchiectasis in a subpleural distribution. Scans were read as "suspicious" for ILAs in the presence of ground-glass, reticular abnormality, diffuse centrilobular nodularity, honeycombing, traction bronchiectasis, non-emphysematous cysts or architectural distortion in at least $5 \%$ of nondependent portions of the lung [18]. Scans with solitary abnormalities that appeared in less than $5 \%$ of the lungs were read as "equivocal" for ILAs, and were included in the group of participants without ILAs. In a sensitivity analysis, these "equivocal" scans were excluded, as previously has been described [10].

\section{High attenuation areas}

HAAs were measured on noncontrast cardiac CT scans performed at the MESA baseline visit and selected follow up examinations using highly standardised protocols [19]. A prior validation study using MESA full lung scans showed that cardiac CT scans image approximately $65-70 \%$ of the total lung volume, capturing most of the lower lobes and excluding much of the lung apexes. Quantitative image attenuation was measured by trained readers using a modified version of the Pulmonary Analysis Software at the University of Iowa Imaging Laboratory (Iowa City, IA, USA) (intraclass correlation 0.93) [9]. An HAA was defined as the percentage of the imaged lung volume having attenuation values between -600 and -250 Hounsfield Units (HU) $[9,10]$. This range of CT lung attenuation includes ground-glass and reticular abnormalities, and excludes denser areas such as atelectasis, medium and large blood vessels and pulmonary nodules. The percentage of emphysema was defined as the percentage of voxels below $-950 \mathrm{HU}$.

\section{Exposure assessment}

Ambient air pollution exposure was estimated for each participant using residential history data (reported from 1980-2012) and spatio-temporal prediction models based on land use regression and geostatistical smoothing. These validated models are described in detail elsewhere [20, 21].

In brief, pollutants were measured at 27 long-term sites, 771 community snapshots and outside 697 participant homes between 2005 and 2009. Using these measurements to supplement pollution monitoring from the monitoring locations in the EPA Air Quality System, MESA Air developed community-specific spatio-temporal models incorporating geographic information and spatial smoothing. These models predict pollutant concentrations at residences for each 2-week period between 1999 and 2012 and explain $90-97 \%$ of the variation in measurements at participant homes.

Given the expectation of a prolonged period of exposure contributing to the development of ILAs, we estimated long-term ambient pollution exposure using 10-year averages of 2-week, residence-specific 
predictions prior to a full lung CT scan. As a sensitivity analysis, we also estimated 5-, 20- and 30-year exposures prior to full lung CT scan. The 20- and 30-year exposures were only available for fine particulate matter (PM2.5; particles with a 50\% cut-off aerodynamic diameter of $2.5 \mu \mathrm{m}$ ) and were based on historic models of annual average exposure developed for periods pre-dating intensive PM monitoring [22]. These long-term residential estimates used address-weighted averages of annual PM2.5 concentration.

To model the progression of HAAs, we estimated long-term exposures as the average of 2-week residence-specific predictions from recruitment to follow-up. Year 2000 concentrations at a participant's address at recruitment were used to model the baseline cross-sectional relationship.

\section{Statistical analyses}

All statistical tests were performed in SAS version 9.3 (SAS Institute, Cary, NC, USA) using a two-tailed $\alpha=0.05$ to define statistical significance.

We used multivariable logistic regression to examine the associations between predicted 10-year pollutant exposures and the odds of ILAs on full lung CT scan at examination 5. Models were adjusted for age, gender, ethnicity, tobacco use (current smoking status and pack years) and site. Sensitivity subanalysis was performed with additional adjustment for occupational exposures, a well-established risk factor for ILD. Occupational exposure was defined according to a previously developed and applied job exposure matrix (JEM) quantifying exposure to vapours, gas, dusts and fumes, but was not available for all participants [23]. Sensitivity analyses were performed using longer exposure periods by averaging 20 - and 30-year estimates of PM2.5 derived from historic model pollution estimates when available. Other studies have shown differential effects on subclinical ILD based on smoking status, ethnicity and gender, so, a priori, we decided to test for effect modification by these variables $[9,11]$. Where stratified models showed different magnitudes of associations, we additionally tested for effect modification with a multiplicative interaction between pollutant exposure and potential effect modifier. We used the likelihood ratio test between the nested main effect and multiplicative models to test for statistical significance.

Linear mixed models were used to analyse the cross-sectional associations between air pollution and HAAs at baseline examination and between air pollution and the rate of progression of HAAs. HAAs were log transformed in the model, and back transformed to obtain estimates of the percentage change. Repeated measurements were modelled as a function of study time, with time-varying exposures modelled as an interaction with time to examine associations between pollutant exposure and the linear rate of change of HAAs over time. Participant-specific random intercepts and slopes were included. Models were adjusted for age, gender, race/ethnicity, educational attainment, JEM, height, body mass index (BMI), waist circumference, smoking status, cigarette pack-years, glomerular filtration rate, total volume of image lung, percentage of emphysema on CT scan, CT scanner type and study site. Potential effect modification was examined by stratification on smoking status, gender and ethnicity.

\section{Results}

A total of 6813 MESA participants had a baseline cardiac CT scan with valid HAA measurements. Of these, 5965 participants had at least one follow-up cardiac CT scan during the study. 470 of these participants were missing either covariate information or pollutant estimates, leaving 5495 participants that were included in the longitudinal analysis of HAA progression (see supplementary figure S1). The mean number of cardiac CT scans per participant was 2.4. The mean follow-up time among the participants with at least two cardiac CT scans was 5.9 years, with a range of 0.9 to 11.4 years.

A total of 3137 MESA participants, including some of the participants recruited under MESA Air, had a full lung CT scan at MESA examination 5 that was assessed by a radiologist. 128 were not read for ILAs and 34 had unreadable scans. In total, 246 participants were missing 10-year pollutant estimates and 58 were missing other covariates, leaving 2671 participants with full lung CT scans in our final analysis of ILAs (see supplementary figure S2). The 491 full lung CT scans that were read as equivocal for ILAs were excluded from the cohort in a sensitivity analysis.

Baseline characteristics and air pollution estimates were similar in the cohorts included in the ILA and HAA analyses (table 1). The mean age of the full cohort at baseline was 62 years (SD 10 years) and 3214 (47\%) were male. Racial/ethnic differences were based on study design: 2621 (39\%) participants were white, 1893 (28\%) were African American, 1496 (22\%) were Hispanic and 803 (11\%) were Chinese American. A total of 3085 (45\%) participants had never smoked and 967 (14\%) were current smokers.

Average air pollution concentration decreased over the study time, as described in detail elsewhere [16]. Mean pollution concentration also varied by site, with the highest levels of PM2.5 in Los Angeles and the highest levels of nitrates (nitrogen oxides $\left[\mathrm{NO}_{\mathrm{x}}\right]$ and nitrogen dioxide $\left[\mathrm{NO}_{2}\right]$ ) in New York City. Pollutant 
TABLE 1 Baseline characteristics of participants included in analyses of subclinical interstitial lung disease (ILD). All parameters were collected at Multi-Ethnic Study of Atherosclerosis (MESA) baseline visits in years 2000-2002 unless otherwise stated

\begin{tabular}{|c|c|c|c|}
\hline Parameter & HAA (examination 1$)^{\#}$ & HAA cohort & ILA cohort" \\
\hline Participants n & 6813 & 5495 & 2671 \\
\hline Age years & $62.2 \pm 10.2$ & $61.6 \pm 10.1$ & $60.1 \pm 9.5$ \\
\hline Male & $3214(47.2)$ & 2703 (49.2) & 1235 (46.2) \\
\hline \multicolumn{4}{|l|}{ Ethnicity } \\
\hline White & 2621 (38.5) & $2238(40.7)$ & 1048 (39.2) \\
\hline African American & $1893(27.8)$ & $1524(27.7)$ & $728(27.3)$ \\
\hline Hispanic & $1496(22)$ & $1141(20.8)$ & 543 (20.3) \\
\hline Asian (Chinese) & 803 (11.8) & $592(10.7)$ & 352 (13.2) \\
\hline BMI $\mathrm{kg} \cdot \mathrm{m}^{-2}$ & $28.3 \pm 5.5$ & $28.4 \pm 5.4$ & $28.3 \pm 5.3$ \\
\hline Height cm & $166.4 \pm 10$ & $166.9 \pm 10$ & $166.7 \pm 10$ \\
\hline Weight lbs & $173.4 \pm 38.2$ & $174.5 \pm 38.0$ & $173.9 \pm 37.7$ \\
\hline \multicolumn{4}{|l|}{ Smoking status } \\
\hline Never smokers & 3085 (45) & 2469 (44.9) & 1494 (55.9) \\
\hline Former smokers & $2761(41)$ & $2261(41.1)$ & 905 (33.9) \\
\hline Current smokers & $967(14)$ & 765 (13.9) & 272 (10.2) \\
\hline Cigarette pack-years & $13(2.0-31.5)$ & $13.5(2.4-31.0)$ & $14.0(4.0-29.6)$ \\
\hline \multicolumn{4}{|l|}{ Education $^{+}$} \\
\hline shigh school & $2460(36.2)$ & 1799 (32.7) & $819(31.4)$ \\
\hline Some college & 1937 (28.5) & $1611(29.3)$ & $749(28.7)$ \\
\hline$\geqslant$ college & 2393 (35.2) & 2085 (37.9) & 1039 (39.9) \\
\hline \multicolumn{4}{|l|}{ Income ${ }^{\S} \$$} \\
\hline$<25000$ & 2059 (31.5) & $1481(27.7)$ & $682(26.9)$ \\
\hline $25000-74999$ & 3003 (45.9) & $2554(47.8)$ & $1156(45.6)$ \\
\hline$\geqslant 75000$ & $1478(22.6)$ & 1309 (24.5) & $700(27.6)$ \\
\hline \multicolumn{4}{|l|}{ Occupational exposure $f$} \\
\hline Low & 4913 (76.2) & $4238(77.1)$ & $1974(78.4)$ \\
\hline Intermediate & $1055(16.4)$ & $860(15.7)$ & $370(14.7)$ \\
\hline High & $481(7.5)$ & $397(7.2)$ & $174(6.9)$ \\
\hline Percent Emphysema & $4.2 \pm 4.5$ & $4.2 \pm 4.4$ & $4.0 \pm 4.0$ \\
\hline \multicolumn{4}{|l|}{ Study site } \\
\hline Winston Salem, NC & $1077(15.8)$ & 899 (16.3) & $490(18.4)$ \\
\hline New York, NY & $1102(16.2)$ & 949 (17.3) & $548(20.5)$ \\
\hline Baltimore, MD & $1086(15.9)$ & 854 (15.5) & $343(12.8)$ \\
\hline St. Paul, MN & $1066(15.7)$ & $862(15.7)$ & 356 (13.3) \\
\hline Chicago, IL & $1164(17.1)$ & 988 (18.0) & $520(19.5)$ \\
\hline Los Angeles, CA & $1318(19.4)$ & $943(17.2)$ & $414(15.5)$ \\
\hline \multicolumn{4}{|l|}{ Pollutants $\# \#$} \\
\hline PM2.5 & $16.8 \pm 2.8$ & $16.7 \pm 2.8$ & $16.5 \pm 2.8$ \\
\hline $\mathrm{NO}_{\mathrm{x}}$ & $50.7 \pm 27.7$ & $50.1 \pm 27.5$ & $49.1 \pm 26.9$ \\
\hline $\mathrm{NO}_{2}$ & $21.5 \pm 9.1$ & $21.3 \pm 9.2$ & $21.0 \pm 9.1$ \\
\hline $\mathrm{O}_{3}$ & $20.2 \pm 4.9$ & $20.3 \pm 4.9$ & $20.4 \pm 5.1$ \\
\hline
\end{tabular}

Data are presented as $\mathrm{n}$, mean $\pm \mathrm{SD}, \mathrm{n}(\%)$ or median (interquartile range) unless otherwise stated. ILA: interstitial lung abnormality; HAA: high attenuation area; BMI: body mass index; PM2.5: particles with a $50 \%$ cut-off aerodynamic diameter of $2.5 \mu \mathrm{m} .{ }^{\#}$ : Excludes one participant without a baseline HAA measurement; ף: Includes demographics for 53 MESA Air new recruits at time of recruitment, with exception of age, which was backdated to $2000 ;^{+}$: Education missing for 23 participants with HAA measured at examination 1 and for 64 participants from the ILA cohort; ${ }^{\S}$ : Income missing for 273 participants with HAA measured at examination 1, for 156 participants from the HAA cohort and for 133 participants from the ILA cohort; $f$ : Exposure to vapours, gas, dust and fumes derived from the job exposure matrix (JEM) missing for 364 participants with HAA measured at examination 1 and for 153 participants from the ILA cohort; \#\#: Year 2000 estimates measured in $\mu \mathrm{g} \cdot \mathrm{m}^{-3}$ for $\mathrm{PM} 2.5$ and in $\mathrm{ppb}$ for other pollutants.

levels were positively correlated with the exception of ozone $\left(\mathrm{O}_{3}\right)$, which was negatively correlated with the other pollutants (see supplementary tables S1 and S2).

Associations of pollutants with interstitial lung abnormalities

$310(9.9 \%)$ of the 3137 participants who underwent full lung CT scan had ILAs: 289 (9.2\%) had scans read as suspicious for ILD and $21(0.67 \%)$ had scans that met standard criteria for a UIP pattern 
with bilateral fibrosis associated with honeycombing and traction bronchiectasis in a subpleural distribution.

In multivariable-adjusted analyses, the odds of ILAs increased 1.77-fold per $40 \mathrm{ppb}$ increment in $\mathrm{NO}_{\mathrm{x}}$ (95\% CI 1.06 to $2.95, \mathrm{p}=0.03$ ). While ILAs were not associated with ambient $\mathrm{PM} 2.5, \mathrm{NO}_{2}$, or $\mathrm{O}_{3}$ concentrations (table 2; figure 1), there was modest evidence of effect modification by smoking status (values for interaction of $\mathrm{p}=0.03$ for $\mathrm{O}_{3}, 0.08$ for $\mathrm{PM} 2.5,0.19$ for $\mathrm{NO}_{\mathrm{x}}$ and 0.28 for $\mathrm{NO}_{2}$ ), with lesser exposure to $\mathrm{O}_{3}$ and greater exposure to $\mathrm{NO}_{\mathrm{x}}$ and $\mathrm{NO}_{2}$, each associated with increased odds of ILAs among never smokers (table 2). For example, each 40-ppb increment in $\mathrm{NO}_{\mathrm{x}}$ was associated with a 2.89-fold greater odds of ILAs (95\% CI 1.35 to $6.16, \mathrm{p}=0.006$ ), while each $10 \mathrm{ppb}$ increment in $\mathrm{O}_{3}$ was associated with an odds ratio for ILAs of 0.30 (95\% CI 0.10 to $0.93, \mathrm{p}=0.04$; table 2 ) among never-smokers.

Sensitivity analyses that 1 ) excluded participants with "equivocal ILA" (see supplementary table S4) or 2) performed additional adjustment for occupational exposures (see supplementary table S5), led to similar findings. Sensitivity analyses with 5-, 20- and 30-year pollutant estimates also yielded similar qualitative results (see supplementary tables S6 and S7).

\section{Associations with high attenuation areas}

At recruitment, MESA participants had a mean HAA of 5.1\% (SD 3.1\%) with a range of $1.4 \%$ to $46.6 \%$. HAAs decreased by an average of $0.35 \%$ (95\% CI 0.21 to $0.48 \%$ ) per year during the study time.

In the primary analysis adjusted for potential confounders (table 3), there was a trend towards HAA progression with $\mathrm{NO}_{\mathrm{x}}$ : a 40 ppb higher $\mathrm{NO}_{\mathrm{x}}$ concentration was associated with a $0.45 \%(95 \% \mathrm{CI}-0.02 \%$ to $0.92 \%, \mathrm{p}=0.06$ ) increase in HAAs per year. Effect estimates for $\mathrm{NO}_{2}$ and $\mathrm{PM} 2.5$ concentrations were in a similar direction.

Race/ethnicity modified the association between pollutant exposure and progression of HAAs for PM2.5, $\mathrm{NO}_{\mathrm{x}}$ and $\mathrm{NO}_{2}$ (values for interaction $\mathrm{p}=0.002,0.007$, and 0.04 , respectively; table 3). For each of these pollutants, the association was strongest in non-Hispanic white people. For example, a $5 \mu \mathrm{g} \cdot \mathrm{m}^{-3}$ increment in $\mathrm{PM} 2.5$ concentration was associated with a $0.93 \%(95 \%$ CI 0.26 to $1.61 \%, \mathrm{p}=0.007)$ increase in HAAs per year in non-Hispanic white people and a $0.45 \%(95 \% \mathrm{CI}-0.70 \%$ to $1.63 \%, \mathrm{p}=0.44)$ increase in HAAs per year in black people. There were no consistent cross-sectional associations between year 2000 pollution estimates and HAA measures in 2000-2002 (supplementary table S3).

\section{Discussion}

We found that exposure to ambient nitrogen oxides was associated with a higher prevalence of ILAs, and, among non-Hispanic white people, exposure to $\mathrm{PM} 2.5, \mathrm{NO}_{\mathrm{x}}$, and $\mathrm{NO}_{2}$ was associated with progression of

TABLE 2 10-year estimates of pollution exposure and risk of interstitial lung abnormalities (ILAs) given as the odds ratio (OR) per concentration increment of the given pollutant

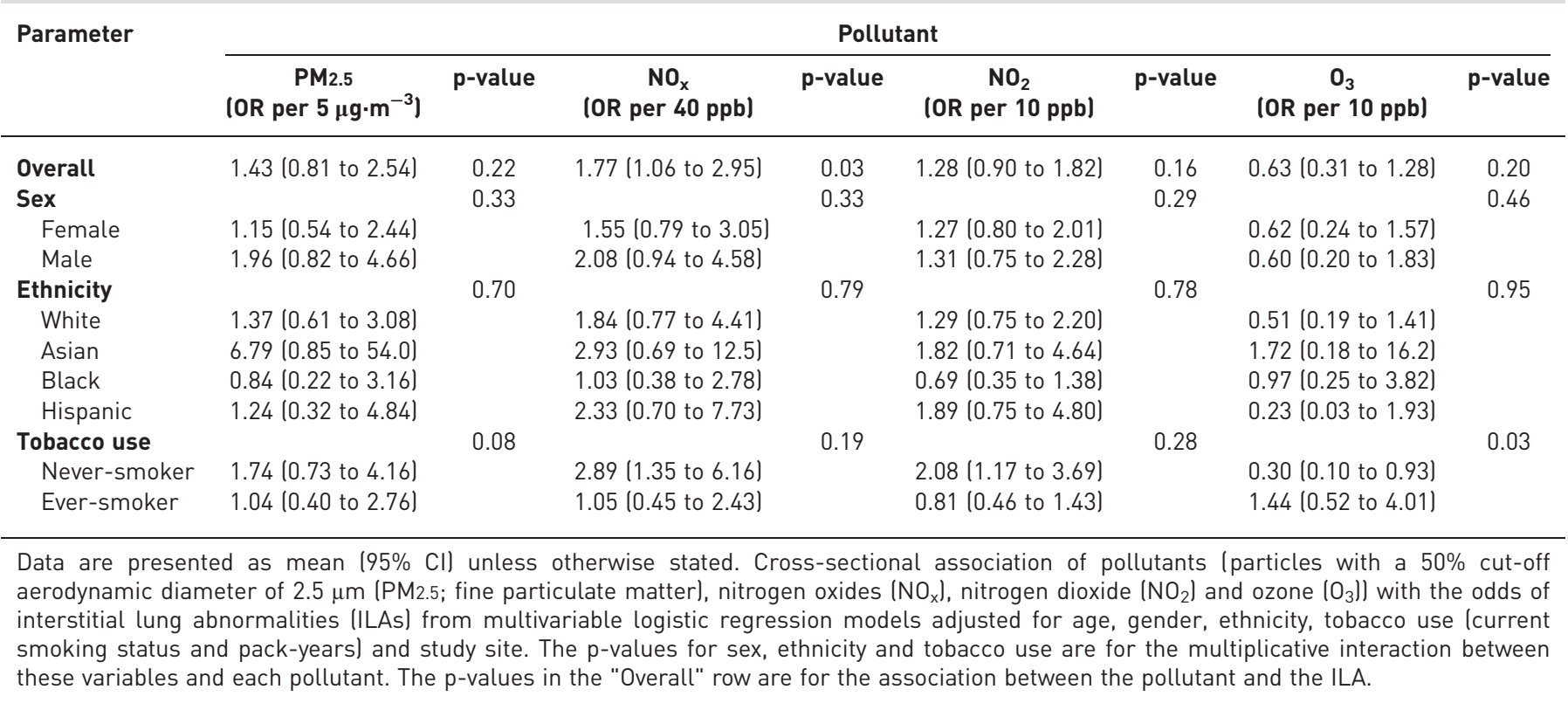



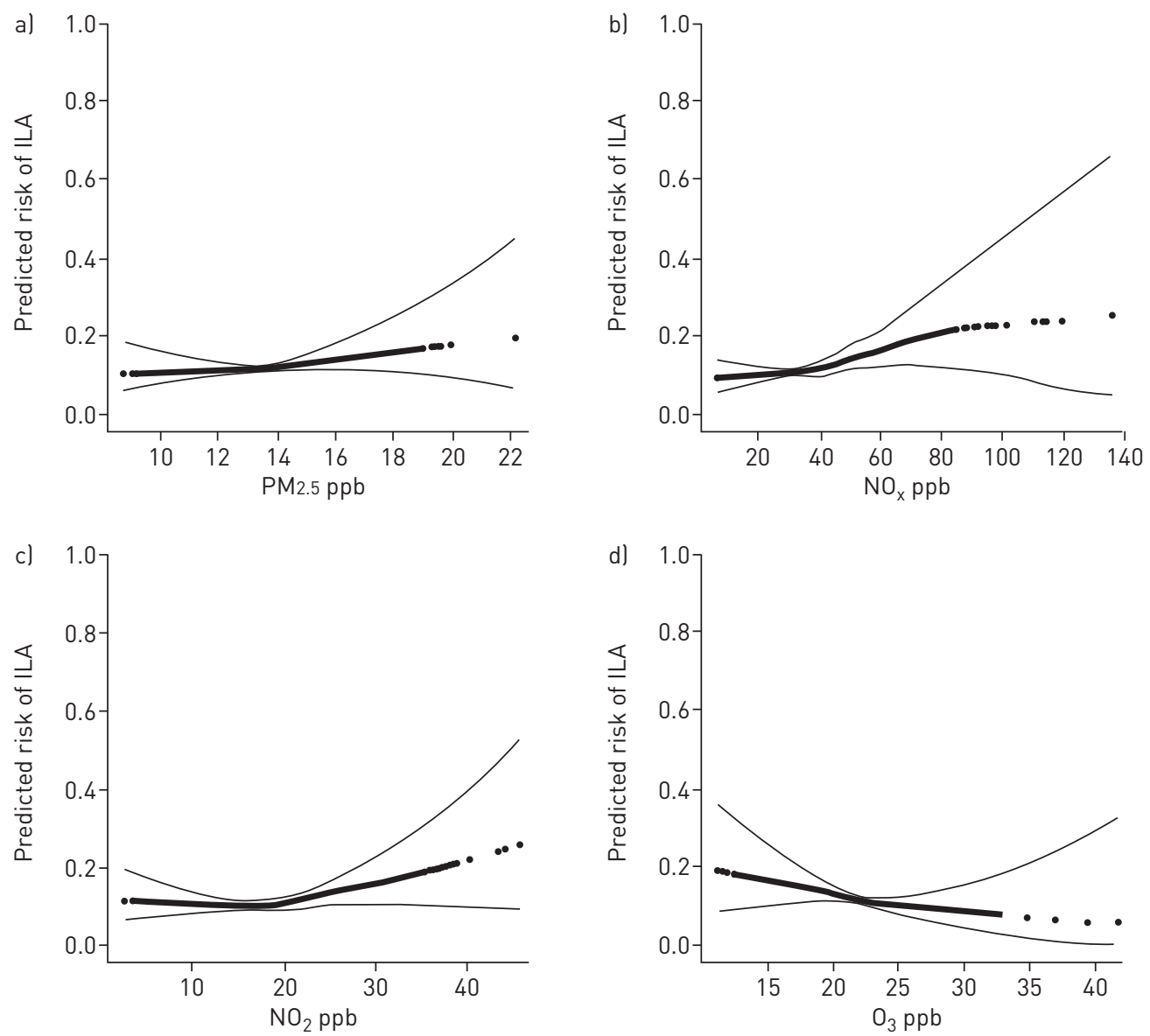

FIGURE 1 Predicted risk of interstitial lung abnormalities (ILAs) with increasing levels of pollutants. a) Particles with a $50 \%$ cut-off aerodynamic diameter of $2.5 \mu \mathrm{m}$ (PM2.5; fine particulate matter), b) nitrogen oxides $\left(\mathrm{NO}_{x}\right), \mathrm{c}$ ) nitrogen dioxide $\left(\mathrm{NO}_{2}\right)$ and d) ozone $\left(\mathrm{O}_{3}\right)$. Effect estimates are derived from general additive models, with points demonstrating the overall effect estimates and lines representing the $95 \%$ confidence bands. All models are adjusted for age, sex, race/ethnicity, tobacco use (pack-years, smoking status) and study site.

HAAs over a median of 5.9 years of follow-up. While less consistent, there was a trend towards an inverse association between greater $\mathrm{O}_{3}$ concentrations and subclinical ILD. This is the first study to link air pollution with early evidence of lung inflammation and fibrosis on CT in community-dwelling adults, adding insight into the hypothesis that air pollution could contribute to ILD.

The associations we found were strongest and most consistent for $\mathrm{NO}_{\mathrm{x}}$, which is predominantly a mixture of nitrogen monoxide (NO) and $\mathrm{NO}_{2}$. These results are similar to those of JoHANNSON et al. [15] in the sole prior study on the impact of air pollution on ILD. In a longitudinal study of 436 patients with idiopathic pulmonary fibrosis (IPF) from Seoul, South Korea, the authors showed that higher levels of $\mathrm{NO}_{2}$ over the previous 6 weeks were associated with increased risk of an IPF exacerbation. Epidemiologic studies have also established the importance of nitrate exposure with other respiratory conditions, including reduced lung function [24], asthma [25] and COPD [26].

$\mathrm{NO}_{\mathrm{x}}$ levels are highest in the near road environment, and may serve as a proxy for a suite of other traffic-related air pollutants (TRAP), including polyaromatic carbons, ultrafine particles and other products of fossil fuel combustion. In comparison, $\mathrm{O}_{3}$ tends to be lower in the near road environment, due both to $\mathrm{O}_{3}$ scavenging by traffic-produced $\mathrm{NO}$ and because $\mathrm{O}_{3}$ is a secondary pollutant, which is photochemically produced in the troposphere by the reaction of sunlight on volatile compounds [27]. This negative correlation could be responsible for the trend towards an inverse effect that we saw with higher levels of $\mathrm{O}_{3}$.

During inhalation, $\mathrm{NO}_{\mathrm{x}}$ and TRAP combine with ammonia and other small particles, to penetrate deep within the bronchial tree. While there is some absorption throughout the respiratory tract, the primary site of injury is at the terminal bronchioles where particles can be phagocytised by airway macrophages, activate pulmonary nocioceptive fibres or cause direct epithelial damage [28]. Mediated by the formation of reactive oxygen species, this leads to airway and systemic inflammation with ongoing cytokine and 
TABLE 3 Long-term estimates of pollution exposure and progression of high attenuation areas (HAAs) given as annual change (\% HAA) per concentration increment of the given pollutant

\begin{tabular}{|c|c|c|c|c|c|c|c|c|}
\hline \multirow[t]{2}{*}{ Parameter } & \multicolumn{8}{|c|}{ Pollutant } \\
\hline & $\begin{array}{l}\text { PM2.5 }(\% \text { HAA } \\
\left.\text { per } 5 \mu \mathrm{g} \cdot \mathrm{m}^{-3}\right)\end{array}$ & p-value & $\begin{array}{l}\mathrm{NO}_{\mathrm{x}}(\% \mathrm{HAA} \\
\text { per } 40 \mathrm{ppb})\end{array}$ & p-value & $\begin{array}{l}\mathrm{NO}_{2}(\% \mathrm{HAA} \\
\text { per } 10 \mathrm{ppb})\end{array}$ & $\mathrm{p}$-value & $\begin{array}{c}\mathrm{O}_{3}(\% \mathrm{HAA} \\
\text { per } 10 \mathrm{ppb})\end{array}$ & p-value \\
\hline Sex & & 0.83 & & 0.43 & & 0.42 & & 0.27 \\
\hline Female & $0.45(-0.25$ to 1.16$)$ & & 0.87 (0.20 to 1.54$)$ & & $0.43(-0.05$ to 0.91$)$ & & $-0.23(-1.07$ to 0.61$)$ & \\
\hline Male & $0.32(-0.40$ to 1.05$)$ & & $-0.05(-0.66$ to 0.27$)$ & & $-0.19(-0.66$ to 0.27$)$ & & $0.57(-0.27$ to 1.41$)$ & \\
\hline Asian & $0.33(-1.07$ to 1.74$)$ & & $0.31(-0.89$ to 1.53$)$ & & $-0.13(-0.88$ to 0.62$)$ & & $1.49(-0.15$ to 3.17$)$ & \\
\hline Black & $0.45(-0.70$ to 1.63$)$ & & $0.80(-0.11$ to 1.71$)$ & & $0.11(-0.60$ to 0.82$)$ & & $0.04(-1.22$ to 1.31$)$ & \\
\hline Hispanic & $0.80(-0.42$ to 2.04$)$ & & $0.34(-0.64$ to 1.38$)$ & & $0.65(-2.10$ to 1.52$)$ & & $-0.97(-2.39$ to 0.46$)$ & \\
\hline Tobacco use & & 0.65 & & 0.46 & & 0.51 & & 0.87 \\
\hline Never-smoker & $0.52(-0.18$ to 1.22$)$ & & $0.66(0.02$ to 1.31$)$ & & $0.33(-0.14$ to 0.80$)$ & & $0.02(-0.83$ to 0.88$)$ & \\
\hline Ever-smoker & $0.46(-0.24$ to 1.18$)$ & & $0.48(-0.17$ to 1.13$)$ & & $0.16(-0.31$ to 0.62$)$ & & $0.07(-0.74$ to 0.89$)$ & \\
\hline
\end{tabular}

Data are presented as mean $(95 \% \mathrm{Cl})$ unless otherwise stated. Linear longitudinal association of pollutants (particles with a $50 \%$ cut-off aerodynamic diameter of $2.5 \mu \mathrm{m}\left(\mathrm{PM} 2.5\right.$; fine particulate matter), nitrogen oxides $\left(\mathrm{NO}_{\mathrm{x}}\right)$, nitrogen dioxide $\left(\mathrm{NO}_{2}\right)$ and ozone $\left.\left(\mathrm{O}_{3}\right)\right)$ with percentage change in high attenuation area (\% HAA) from linear mixed models adjusted for age, sex, race/ethnicity, educational attainment, job exposure matrix (JEM), height, body mass index (BMI), waist circumference, smoking status, cigarette pack-years, glomerular filtration rate, total volume of image lung, percent emphysema on computed tomography (CT) scan, CT scanner type and study site. The p-values for sex, ethnicity and tobacco use are for the multiplicative interaction between these variables and each pollutant. The p-values in the "Overall" row are for the association between the pollutant and HAA.

chemokine release. Murine models have demonstrated that nitrate exposure causes cellular injury, epithelial proliferation, hyperplasia, inflammation and fibrotic changes [29]. In genetically susceptible humans, this could potentially initiate or contribute to the cycle of alveolar injury, disordered repair and fibrosis seen in the ILDs.

In this study, ethnicity modified the impact of pollution exposure, with the strongest effect on HAA progression in non-Hispanic white people. Findings from prior air pollution studies have been mixed, with studies showing smaller, larger or similar effect sizes in different ethnic and racial groups [30]. Our results may indicate that the detrimental effects from unmeasured healthcare disparities and other confounders may overwhelm the adverse consequences of ambient air pollution exposure. A similar phenomenon may drive the suggestion of the differential effect that we observed with smoking status, where the association may be greatest in nonsmokers [31]. Alternatively, there are significant differences in the prevalence of some subtypes of ILD by ethnic group, which may account for the different effects of air pollution we observed.

This was an observational study with inherent limitations of exposure and outcome misclassification, residual confounding and the inability to infer causation. Our definition of subclinical ILD was based on radiologic findings rather than histologic changes. It is possible that our quantitative measurement of lung density included other pulmonary processes, such as oedema, infection or atelectasis. However, as described in our prior studies of this cohort, there was no evidence of confounding by cardiac function, obesity or the percentage of lung imaged [10]. In addition, changes in scanning technology through the study period may introduce confounding which is difficult to control for in analyses.

Although our models adjusted for a variety of confounders, there may have been residual confounding from unmeasured variables, such as other environmental factors (e.g. moulds, avian antigens, etc.).

While our exposure estimates predicted ambient pollutant concentrations at participants' residences, they may not accurately reflect pollutant exposure in the participants' microenvironment. The internalised dose of pollution in an individual may vary widely depending on multiple variables, such as the time spent outdoors, hobbies, physical activity, commuting and vacations.

The longer-term estimates of particulate matter exposure (20 and 30 years) that were derived from historic models may be less accurate than our other pollutant estimates, which did not extrapolate to time periods with limited data and also benefitted from spatially intensive air pollution monitoring data. This 
non-differential exposure misclassification would be expected to weaken the strength of associations with ILAs and HAAs for the longer exposure periods.

Despite these limitations, the effects we observed represent important subclinical changes in the lungs of asymptomatic community-dwelling US adults. We used sophisticated modelling to produce individual estimates of long-term exposure to air pollution, and investigated the effect of different lag times of exposure. By adjusting for site, we were able to eliminate unmeasured confounders between cities and focus on within city variations in pollutant levels.

In summary, we found that ILA was associated with long-term average exposure to oxides of nitrogen; and in non-Hispanic white people, HAA progression was associated with exposure to both oxides of nitrogen, nitrogen dioxide and fine particulates. More epidemiologic and translational studies will be needed to investigate the roles of individual pollutants and identify the potential biological pathways underlying these associations. Although definitive evidence is still missing, this study provides supporting evidence that air pollution could be a potential risk factor for ILD. If proven, this would have important clinical implications for patients and serve to help inform public policy.

\section{Acknowledgements}

The authors thank the other investigators, the staff and the participants of the MESA study for their valuable contributions. A list of participating MESA investigators and institutions can be found at www.mesa-nhlbi.org. The coding of occupational information was conducted by the National Institute for Occupational Safety and Health (NORA FY08 CRN SLB8).

All authors contributed substantially to the conception and design of the manuscript and the acquisition, analysis, or interpretation of data. All authors contributed to drafting the manuscript or critical revision for important intellectual content. All authors approved the final submitted version.

\section{References}

Guarnieri M, Balmes JR. Outdoor air pollution and asthma. Lancet 2014; 383: 1581-1592.

2 Adar SD, Kaufman JD, Diez-Roux AV, et al. Air pollution and percent emphysema identified by computed tomography in the multi-ethnic study of atherosclerosis. Environ Health Perspect 2015; 123: 144-151.

3 Goss CH, Newsom SA, Schildcrout JS, et al. Effect of ambient air pollution on pulmonary exacerbations and lung function in cystic fibrosis. Am J Respir Crit Care Med 2004; 169: 816-821.

4 Rice MB, Rifas-Shiman SL, Oken E, et al. Exposure to traffic and early life respiratory infection: a cohort study. Pediatr Pulmonol 2015; 50: 252-259.

5 Bhinder S, Chen H, Sato M, et al. Air pollution and the development of posttransplant chronic lung allograft dysfunction. Am J Transplant 2014; 14: 2749-2757.

6 Turner MC, Krewski D, Pope CA 3rd, et al. Long-term ambient fine particulate matter air pollution and lung cancer in a large cohort of never-smokers. Am J Respir Crit Care Med 2011; 184: 1374-1381.

7 Raghu G, Chen SY, Yeh WS, et al. Idiopathic pulmonary fibrosis in US Medicare beneficiaries aged 65 years and older: incidence, prevalence, and survival, 2001-11. Lancet Respir Med 2014; 2: 566-572.

8 Doyle TJ, Hunninghake GM, Rosas IO. Subclinical interstitial lung disease: why you should care. Am J Respir Crit Care Med 2012; 185: 1147-1153.

9 Lederer DJ, Enright PL, Kawut SM, et al. Cigarette smoking is associated with subclinical parenchymal lung disease: the Multi-Ethnic Study of Atherosclerosis (MESA)-lung study. Am J Respir Crit Care Med 2009; 180: 407-414.

10 Podolanczuk AJ, Oelsner EC, Barr RG, et al. High attenuation areas on chest computed tomography in community-dwelling adults: the MESA study. Eur Respir J 2016; 48: 1442-1452.

11 Johnson C, Giles JT, Bathon J, et al. Smoking and subclinical ILD in RA versus the Multi-Ethnic Study of Atherosclerosis. PloS One 2016; 11: e0153024.

12 Araki T, Putman RK, Hatabu H, et al. Development and progression of interstitial lung abnormalities in the Framingham Heart Study. Am J Respir Crit Care Med 2016; 194: 1514-1522.

13 Putman RK, Hatabu H, Araki T, et al. Association between interstitial lung abnormalities and all-cause mortality. JAMA 2016; 315: 672-681.

14 Doyle TJ, Washko GR, Fernandez IE, et al. Interstitial lung abnormalities and reduced exercise capacity. Am J Respir Crit Care Med 2012; 185: 756-762.

15 Johannson KA, Vittinghoff E, Lee K, et al. Acute exacerbation of idiopathic pulmonary fibrosis associated with air pollution exposure. Eur Respir J 2014; 43: 1124-1131.

16 Kaufman JD, Adar SD, Barr RG, et al. Association between air pollution and coronary artery calcification within six metropolitan areas in the USA (the Multi-Ethnic Study of Atherosclerosis and Air Pollution): a longitudinal cohort study. Lancet 2016; 388: 696-704.

17 Sieren JP, Newell JD Jr, Barr RG, et al. SPIROMICS protocol for multicenter quantitative computed tomography to phenotype the lungs. Am J Respir Crit Care Med 2016; 194: 794-806.

18 Washko GR, Hunninghake GM, Fernandez IE, et al. Lung volumes and emphysema in smokers with interstitial lung abnormalities. N Engl J Med 2011; 364: 897-906.

19 Hoffman EA, Jiang R, Baumhauer $\mathrm{H}$, et al. Reproducibility and validity of lung density measures from cardiac CT scans-the Multi-Ethnic Study of Atherosclerosis (MESA) lung study. Acad Radiol 2009; 16: 689-699.

20 Bergen S, Sheppard L, Sampson PD, et al. A national prediction model for PM2.5 component exposures and measurement error-corrected health effect inference. Environ Health Perspect 2013; 121: 1017-1025.

21 Keller JP, Olives C, Kim SY, et al. A unified spatiotemporal modeling approach for predicting concentrations of multiple air pollutants in the multi-ethnic study of atherosclerosis and air pollution. Environ Health Perspect 2015; 123: 301-309. 
22 Kim SY, Olives C, Sheppard L, et al. Historical prediction modeling approach for estimating long-term concentrations of PM2.5 in cohort studies before the 1999 implementation of widespread monitoring. Environ Health Perspect 2017; 125: 38-46.

23 Doney B, Hnizdo E, Graziani M, et al. Occupational risk factors for COPD phenotypes in the Multi-Ethnic Study of Atherosclerosis (MESA) lung study. COPD 2014; 11: 368-380.

24 Mölter A, Agius RM, de Vocht F, et al. Long-term exposure to $\mathrm{PM}_{10}$ and $\mathrm{NO}_{2}$ in association with lung volume and airway resistance in the MAAS birth cohort. Environ Health Perspect 2013; 121: 1232-1238.

25 Mann JK, Balmes JR, Bruckner TA, et al. Short-term effects of air pollution on wheeze in asthmatic children in Fresno, California. Environ Health Perspect 2010; 118: 1497-1502.

26 Faustini A, Stafoggia M, Cappai G, et al. Short-term effects of air pollution in a cohort of patients with chronic obstructive pulmonary disease. Epidemiology (Cambridge, Mass) 2012; 23: 861-879.

27 Brunekreef B, Holgate ST. Air pollution and health. Lancet 2002; 360: 1233-1242.

28 Akopian AN, Fanick ER, Brooks EG. TRP channels and traffic-related environmental pollution-induced pulmonary disease. Semin Immunopathol 2016; 38: 331-338.

29 Persinger RL, Poynter ME, Ckless K, et al. Molecular mechanisms of nitrogen dioxide induced epithelial injury in the lung. Mol Cell Biochem 2002; 234-235: 71-80.

30 Hicken MT, Adar SD, Hajat A, et al. Air pollution, cardiovascular outcomes, and social disadvantage: the multiethnic study of atherosclerosis. Epidemiology 2016; 27: 42-50.

31 Schultz ES, Hallberg J, Bellander T, et al. Early-life exposure to traffic-related air pollution and lung function in adolescence. Am J Respir Crit Care Med 2016; 193: 171-177. 